\title{
Galeazzi Lesions in Children and Adolescents
}

\section{Treatment and Outcome}

\author{
Robert Eberl MD, Georg Singer MD, \\ Johannes Schalamon MD, Thomas Petnehazy MD, \\ Michael E. Hoellwarth MD
}

Received: 6 December 2007 / Accepted: 9 April 2008/Published online: 29 April 2008

(C) The Association of Bone and Joint Surgeons 2008

\begin{abstract}
A Galeazzi fracture is defined as a fracture of the radius associated with dislocation of the distal radioulnar joint. Treatment in children and adolescents is usually possible with closed reduction and casting. The objective of this retrospectively designed study was to describe all Galeazzi lesions treated at our department during a 3-year period. One hundred ninety-eight patients with displaced fractures of the radius alone or both bones of the forearm were reviewed. In 26 (13\%) cases, a Galeazzi lesion was found and these patients formed the study group. Outcome was assessed using the GartlandWerley score. Eight of $26(31 \%)$ fractures were recognized initially and classified as a Galeazzi lesion. Casting after fracture reduction was possible in 22 patients. Thirteen patients were treated with immobilization in a below-elbow cast and nine with an above-elbow cast. Four patients were treated operatively. The results were excellent in 23 cases and good in three cases. In cases of distal forearm fractures, a possible Galeazzi lesion should be considered. However, proper reduction of the radius with concomitant reduction of the distal radioulnar joint and cast immobilization
\end{abstract}

Each author certifies that he or she has no commercial associations (eg, consultancies, stock ownerships, equity interest, patent/licensing arrangements, etc) that might pose a conflict of interest in connection with the submitted article.

Each author certifies that his or her institution either has waived or does not require approval for the human protocol for this investigation and that all investigations were conducted in conformity with ethical principles of research.

R. Eberl ( $)$, G. Singer, J. Schalamon, T. Petnehazy,

M. E. Hoellwarth

Department of Pediatric Surgery, Medical University of Graz,

Auenbruggerplatz 34, 8036 Graz, Austria

e-mail: robert.eberl@meduni-graz.at provides good to excellent outcome even if the Galeazzi lesion is primarily not recognized.

Level of Evidence: Level IV, therapeutic study. See the Guidelines for Authors for a complete description of levels of evidence.

\section{Introduction}

Children and adolescents frequently present to emergency departments with fractures of the forearm [17]. The Galeazzi fracture, which is named after the Italian surgeon Riccardo Galeazzi, was first described by Cooper in 1822, according to Voigt and Lill [18]. Representing a special case of forearm fractures, it is classified as a fracture of the radius at any level associated with disruption of the distal radioulnar joint (DRUJ) and resulting luxation of the ulna [19]. The typical mechanism of injury is described as a fall on an outstretched hand in hyperpronation [14].

In adults, this fracture occurs with an incidence of $2.7 \%$ to $6.8 \%$ of all forearm fractures $[8,18]$. In children, reports regarding the incidence of this type of injury differ substantively from $0.3 \%$ to $2.8 \%[17,19]$. The peak incidence occurs between the ages of 9 and 13 years [19]. Galeazzi lesions frequently are underdiagnosed. In the only reported series of pediatric patients with this type of fracture, $41 \%$ of Galeazzi lesions were unrecognized [19]. However, there is lack of additional information in the recent literature about the rate of undiagnosed Galeazzi lesions in children.

Standard treatment in adults consists of operative intervention with plate fixation and in some cases temporary transfixation of the DRUJ using Kirschner wires [2, 5, 9]. In the pediatric population, good results after closed reduction and casting have been reported $[10,19]$. The gold 
standard of conservative treatment in children is aboveelbow casting in supination [15]. Conservative treatment of Galeazzi fractures has been shown to lead to unsatisfactory results of as much as $92 \%$ in adults. In contrast, the final results of conservative treatment are generally good in children [19]. The only published report focusing on Galeazzi fractures in children shows the outcome in children treated with a below-elbow cast is worse compared with the group treated with above-elbow casting [19]. Additionally, there is lack of information regarding how the initial misdiagnosis of a Galeazzi lesion influences treatment and outcome in the pediatric population.

Therefore, the main objective of our retrospective study was to analyze the incidence of Galeazzi fractures in children with a dislocated fracture of the forearm and to report the percentage of misdiagnosed Galeazzi fractures in this group of patients. Additionally, we sought to report the proportion of conservative versus operative treatments in pediatric Galeazzi lesions. To verify the treatment strategies, the outcome of all children and adolescents treated with Galeazzi fractures was assessed.

\section{Materials and Methods}

We retrospectively analyzed the medical data of all children who were admitted for inpatient treatment after fractures of the forearm. Our local policy requires inpatient treatment in cases of major dislocated fractures, which require manipulation. Minimally displaced fractures were reduced using conscious sedation at the outpatient department and the patients were excluded from the study. From 2004 to 2006, 198 children with displaced forearm fractures were identified. At admission, information was obtained regarding the mechanism of injury. Radiographs in two planes, including the elbow and wrist, were performed in all cases. As part of this study, these radiographs were revisited to identify all children with Galeazzi lesions and were viewed by three of the authors (RE, GS, JS) who were blinded to the results. A Galeazzi lesion was defined as a fracture of one or both bones of the forearm at any level in combination with a dislocation of the DRUJ. Localization of these fractures and displacement of the ulna were recorded. Additionally, the initial medical reports were reviewed to determine whether the nature of the injury was recognized at first attendance.

With the children under general anesthesia, closed reduction was performed and plaster casts were applied for immobilization of the fractures. In case of irreducibility or persistent instability, operative treatment was performed with plate osteosynthesis or intramedullary nailing depending on the fracture localization. None of the authors was the operating surgeon. Followups, including radiographs, were performed 1 and 3 weeks after discharge and at the time of final medical examination. The duration of plaster cast immobilization was chosen according to the radiologic signs of bone healing. Implant removal was performed 6 to 9 months after implantation.

All patients were reexamined 8 to 39 months (mean, 22 months) after trauma by one observer (TP) to complete the Gartland-Werley score [4]. This score is based on a rating system considering residual deformity, pain, range of motion, and complications during treatment. Results are classified as excellent ( $0-2$ points), good (3-8 points), fair (9-20 points), or poor (21 points or more). Data were entered in a Microsoft Excel (Microsoft Inc, Redmond, WA) spreadsheet for additional analysis. Because of the relatively small numbers of patients, this study is a descriptive report and therefore statistical analysis was not performed. According to the guidelines of the local ethics committee, informed written consent was not required for this retrospective study.

\section{Results}

We identified 26 Galeazzi lesions in 198 patients (13\%) who were admitted with dislocated forearm fractures; eight of those 26 fractures (31\%) initially were recognized and classified as Galeazzi lesions. Six of these patients (3\%) had fractures of both bones of the forearm, whereas the remaining 20 patients $(10 \%)$ presented with an isolated fracture of the radius in combination with DRUJ involvement. Seventeen $(65 \%)$ patients were male and nine $(35 \%)$ were female. The average age of the patients with a Galeazzi lesion was 11 years (range, $4.5-16$ years). The average age of the patients without a Galeazzi lesion was similar at 12.2 years in boys and 11.9 years in girls. None of our patients could provide reliable information regarding the exact mechanism of injury. The majority of our patients had dorsal dislocation of the ulna $(\mathrm{n}=19$ [73\%]) associated either with an isolated fracture of the radius in palmar position or fractures of both bones of the forearm. Seven patients $(27 \%)$ presented with palmar dislocation of the ulna (Table 1). All fractures were located in the distal third of the radius or at the border of the middle to the distal thirds of the forearm. Neither a reverse Galeazzi lesion nor

Table 1. Distribution of the dislocation of the ulna in 26 Galeazzi fractures

\begin{tabular}{lll}
\hline Dislocation of the ulna & \multicolumn{2}{l}{ Fractured bone } \\
\cline { 2 - 3 } & Radius & Radius and ulna \\
\hline Dorsal & 13 & 6 \\
Palmar & 7 & 0 \\
\hline
\end{tabular}



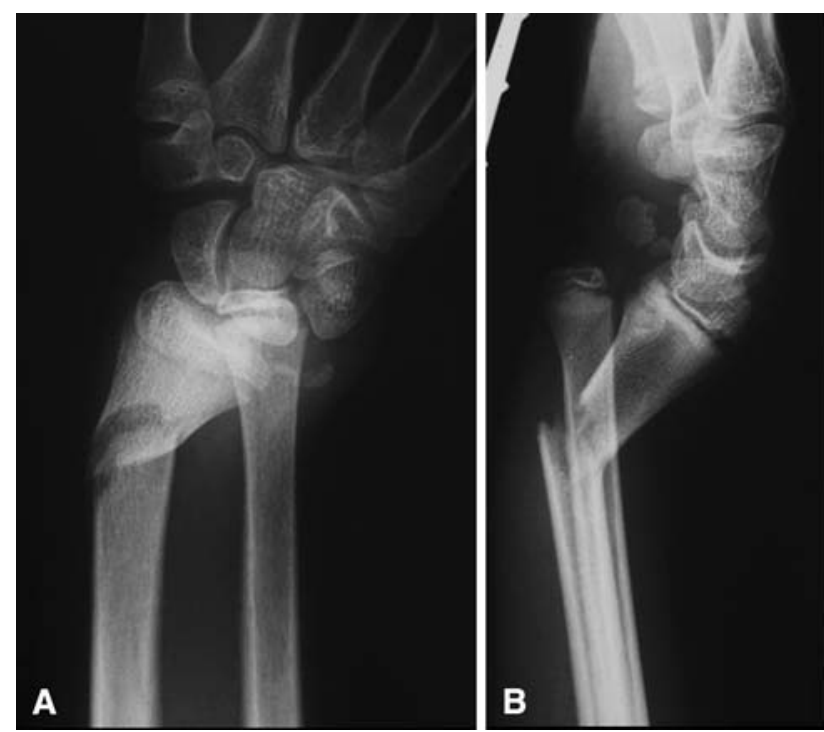

Fig. 1A-B In a 14-year-old boy with a Galeazzi fracture of the right arm, an (A) AP view and (B) lateral view at the time of admission show palmar dislocation of the ulna and a fracture of the radius in the distal third.

a Galeazzi-equivalent lesion with disruption of the distal ulnar epiphysis was found in our group of patients.

Although closed reduction and casting were possible in 22 patients $(85 \%)$, only four patients $(15 \%)$ were treated operatively. Reduction was performed with the patients under general anesthesia in all cases. Immobilization with an above-elbow plaster cast for 6 weeks was required in nine cases (35\%). This group consisted of eight patients with an initially diagnosed Galeazzi injury (cast in full supination) and one patient with a Galeazzi lesion after fracture of the radius and ulna, which was misdiagnosed as a fracture of the forearm and recognized at the time of this review (cast in neutral position). Thirteen patients (50\%) wore a below-elbow plaster cast (Figs. 1, 2). Another four children required operative stabilization; two children $(8$ and 9 years old) were treated with intramedullary elastic titanium nails using retrograde nailing of the radius and antegrade nailing of the ulna. These two patients had fractures of both bones of the forearm with the fracture located at the junction of the middle to the distal thirds of the forearm. The Galeazzi lesion was not recognized at admission but was recognized during surgery. In the remaining two cases, dorsal plate fixation of the radius was required. In both cases, the lesion was not recognized at admission or during surgery. A 15-year-old girl sustained a refracture of the radius and an additional fracture and dislocation of the ulna. A 16-year-old boy presented 1 week after initial admission with secondary displacement and loss of reduction in the DRUJ despite initial anatomic alignment and above-elbow cast immobilization. The loss

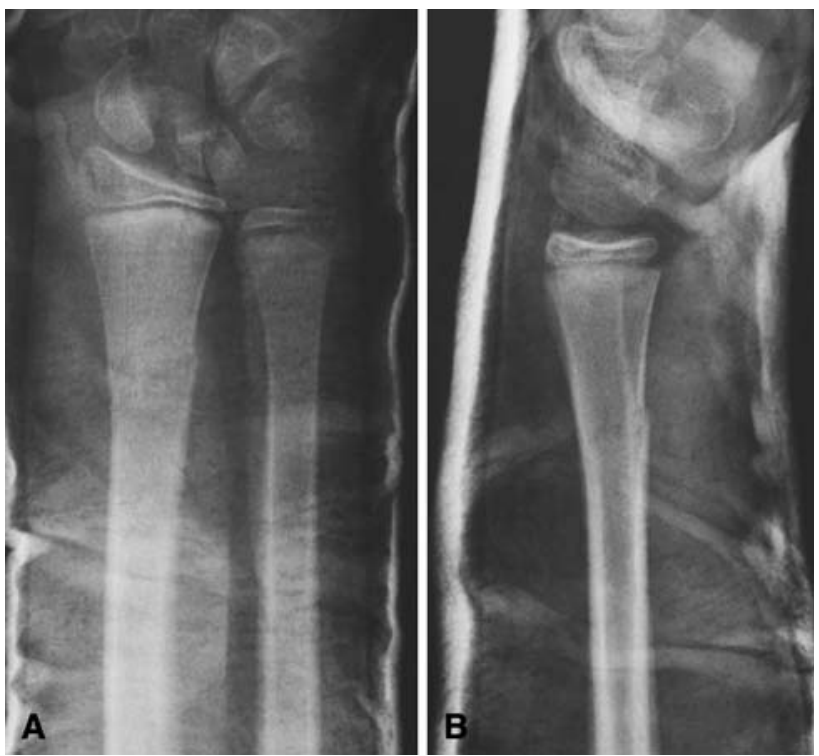

Fig. 2A-B (A) An AP view and (B) lateral view are shown immediately after repositioning and plaster casting of the same patient as in Figure 1. The casting was done with a below-elbow cast.

of reduction in the DRUJ was treated effectively with plate osteosynthesis.

At followup, 23 patients were symptom-free. Two children reported occasional pain and weakness in the wrist; another child had slight limitation of motion of the thumb after rupture of the long extensor tendon. The DRUJ was stable at followup in all children. The result using the Gartland-Werley score was excellent in 23 cases and good in three cases. Children with below-elbow casts had better results (Table 2). One child experienced a complicated clinical course. As a consequence of intramedullary fracture stabilization, the patient had hypoesthesia in the area of the superficial radial nerve over the first extensor compartment. On the 69 th postoperative day, rupture of the tendon of the extensor pollicis longus muscle occurred after minimal trauma. This rupture was caused by a prominent and sharp nail end close to the tendon. The patient required transfer of the tendon of the extensor

Table 2. Outcome related to mode of treatment and initial recognition of the Galeazzi fracture*

\begin{tabular}{lccllrr}
\hline Treatment & \multicolumn{2}{c}{ Outcome } & & \multicolumn{2}{l}{ Correct diagnosis } \\
\cline { 2 - 3 } & Excellent & Good & & Yes & No \\
\hline Above-elbow cast & 7 & 2 & & 8 & 1 \\
Below-elbow cast & 13 & 0 & & 0 & 13 \\
Internal fixation & 3 & 1 & & 0 & 4 \\
\hline
\end{tabular}

* The outcome was excellent and very good even with unrecognized Galeazzi fractures and the majority of correctly identified Galeazzi fractures was treated with an above-elbow cast. 
indicis muscle. However, the patient had slight limitation of range of motion (loss of $10^{\circ}$ for dorsal extension, no loss in pronation or supination) and persistent hypoesthesia at followup 16 months after trauma.

\section{Discussion}

In our series of 198 skeletally immature children with a displaced fracture of the forearm, a Galeazzi lesion was identified in 26 children (13\%). Although others have described a frequency less than $5 \%$ of disruptions of the DRUJ, in children, the rate of overlooked Galeazzi lesions may be much higher [19]. Concordant with Walsh et al. [19] the median age of the patients in our series was 11 years with the majority being male. In addition, it is accepted that the radius fracture accompanying the Galeazzi lesion is located most often at the junction of the middle and distal thirds of the bone [12]. Our data confirm these findings with all of the fractures being located in the distal third of the radius.

Unfortunately, our patients could not describe the exact mechanism of injury. All remembered falling on the hand but could not remember the exact position of the hand at the time of impact. Although Frykman [3] produced this fracture experimentally with axial loading alone, others $[14,18]$ assume this fracture is a combination of hyperpronation and a fall on the outstretched hand. We propose the different directions of displacement are caused by different mechanisms of injuries; a posterior displacement of the ulna is caused by a longitudinal force on the arm in supination, whereas anterior displacement is more likely if the hand is in a pronated position.

A possible reason for the considerable rate of overlooked Galeazzi lesions may be a lack of information regarding the specific classification of fractures in the distal forearm. Although the majority (18 of 26 [69\%]) of the retrospectively classified Galeazzi lesions were not primarily described in our series, displacement of the ulna was recognized and corrected in all children, and persistent displacement of the DRUJ after initial reduction could not be found. Because of this observation, we do not recommend conducting a thorough examination of the distal radioulnar joint before reduction of the forearm [5]. A clinical examination to identify possible instability before reduction has no consequences. Additionally, the clinical examination in case of a fracture is painful and should be performed with the patient under general anesthesia to achieve objective information. In case of irreducibility, manipulation under general anesthesia is required to exclude possible soft tissue impingement. If soft tissue impingement is suspected, an open procedure for exploration of the joint and open reduction and internal fixation are mandatory.
In adults, Galeazzi fractures usually require operative treatment. Numerous authors stated a considerable rate of secondary displacements and nonunions may result in case of conservative treatment $[7,10,13,16]$. Voigt and Lill and Mikic assumed disruption of the distal triangular fibrocartilage complex leads to persistent instability of the DRUJ because muscle forces may not be controlled by cast immobilization only $[10,18]$. Open reduction with fixation of the radial fracture using plates and screws, transfixation of the DRUJ, and immobilization with an above-elbow plaster cast led to good functional results and therefore is recommended as the preferred treatment in adult patients $[10,13,16]$. In contrast to the adult population, the treatment of Galeazzi lesions in children usually has a good outcome after conservative treatment with cast immobilization [16]. Fractures in children are different from adulttype fractures. Incomplete fractures, bowing fractures, and intact periosteal structures provide residual stability after bone trauma. In addition, the increased elasticity of the ligamental structures in children may compensate for displacement of the ulna and prevent ligamental rupture in children with Galeazzi injuries [1]. However, we could not find studies of soft tissue imaging of the acute posttraumatic anatomy of the DRUJ to identify or exclude ligamental injury.

The considerably higher rate of Galeazzi fractures in our series may be attributed to the selection bias (we included only patients with significant displacement and inpatient treatment). Other limitations of our study are the retrospective design and the relatively small number of patients. Furthermore, we did not have a clear strategy for immobilization of Galeazzi fractures; therefore, the decision to use below- or above-elbow casts followed the preferences of the treating surgeon. However, if a Galeazzi lesion was identified at first attendance, all surgeons decided to use above-elbow casts for immobilization according to the recommended guidelines [1]. Therefore, all surgeons attempted to achieve correct alignment in the wrist. Half of the fractures in our series were underdiagnosed and immobilized in a below-elbow cast with an excellent outcome in all cases. This may be attributed to the fact that early functional treatment was possible. After anatomic reduction of the DRUJ, a well-shaped below-elbow cast allows pronation and supination and prevents redislocation of the joint, whereas the majority of reports in the medical literature describing Galeazzi fractures in children prefer treatment with above-elbow casts [6, 11]. In older children and adolescents with considerable dislocation and instability despite cast application, operative fracture stabilization may become necessary. Above-elbow casts are useful with fractures of both bones of the forearm but are not required in typical Galeazzi injuries. However, it is possible the better results related to below-elbow casts may 
be attributed to selection bias and that the patients with above-elbow casts in our series had more severe injuries. Malunion of the radius, subluxation in the radioulnar joint with resulting overgrowth, injury to the ulnar nerve, or limited range of motion for pronation and supination, which represent common complications of Galeazzi fractures [16], were not found in our study group. All longterm problems either were caused by technical problems (rupture of the tendon by a sharp nail end) or potentially were unpreventable (occasional pain and weakness in the wrist). However, long-term instability of the DRUJ was not observed, thereby justifying our treatment approach.

Distal fractures of the forearm in children require exact radiologic imaging and adequate diagnosis to recognize a possible Galeazzi lesion. Especially with markedly displaced forearm fractures, a Galeazzi lesion should be considered by the treating surgeon. Proper reduction of the radius with subsequent reduction of the ulna in the DRUJ and cast immobilization provide good to excellent outcomes even if the Galeazzi lesion is primarily underdiagnosed. Long-term instability of the DRUJ after Galeazzi lesions was not observed in our series of pediatric patients.

\section{References}

1. Barros EM, Rodrigues CJ, Rodrigues NR, Oliveira RP, Barros TE, Rodrigues AJ Jr. Aging of the elastic and collagen fibers in the human cervical interspinous ligaments. Spine J. 2002;2: 57-62.

2. Bhan S, Rath S. Management of the Galeazzi fracture. Int Orthop. 1991;15:193-196.

3. Frykman G. Fracture of the distal radius including sequelae: shoulder-hand-finger syndrome, disturbance in the distal radio- ulnar joint and impairment of nerve function: a clinical and experimental study. Acta Orthop Scand. 1967;Suppl 108:3.

4. Gartland JJ Jr, Werley CW. Evaluation of healed Colles' fractures. J Bone Joint Surg Am. 1951;33:895-907.

5. Giannoulis FS, Sotereanos DG. Galeazzi fractures and dislocations. Hand Clin. 2007;23:153-163, v.

6. Hostetler MA, Davis CO. Galeazzi fracture resulting from electrical shock. Pediatr Emerg Care. 2000;16:258-259.

7. Hughston JC. Fracture of the distal radial shaft: mistakes in management. J Bone Joint Surg Am. 1957;39:249-264, passim.

8. Lechner J, Steiger R, Ochsner P. [Surgical treatment of Galeazzi fracture][in German]. Unfallchirurg. 1993;96:18-23.

9. Macule Beneyto F, Arandes Renu JM, Ferreres Claramunt A, Ramon Soler R. Treatment of Galeazzi fracture-dislocations. J Trauma. 1994;36:352-355.

10. Mikic ZD. Galeazzi fracture-dislocations. J Bone Joint Surg Am. 1975;57:1071-1080.

11. Ooi LH, Toh CL. Galeazzi-equivalent fracture in children associated with tendon entrapment: report of two cases. Ann Acad Med Singapore. 2001;30:51-54.

12. Perron AD, Hersh RE, Brady WJ, Keats TE. Orthopedic pitfalls in the ED: Galeazzi and Monteggia fracture-dislocation. Am J Emerg Med. 2001;19:225-228.

13. Reckling FW. Unstable fracture-dislocations of the forearm (Monteggia and Galeazzi lesions). J Bone Joint Surg Am. 1982;64:857-863.

14. Reckling FW, Cordell LD. Unstable fracture-dislocations of the forearm: the Monteggia and Galeazzi lesions. Arch Surg. 1968;96:999-1007.

15. Rodriguez-Merchan EC. Pediatric fractures of the forearm. Clin Orthop Relat Res. 2005;432:65-72.

16. Rothe M, Rudy T, Stankovic P, Sturmer KM. [Treatment of Galeazzi's fracture: is the surgical revision of the distal radioulnar joint necessary?] [in German]. Handchir Mikrochir Plast Chir. 2001;33:252-257.

17. Schlickewei W, Oberle M. [Forearm fractures in children][in German]. Unfallchirurg. 2005;108:223-232.

18. Voigt C, Lill H. Combined fractures of the forearm: Monteggia, Galeazzi and Essex-Lopresti lesions. Aktuelle Traumatol. 2004;34:270-277.

19. Walsh HP, McLaren CA, Owen R. Galeazzi fractures in children. J Bone Joint Surg Br. 1987;69:730-733. 